DOI: 10.17516/1997-1397-2020-13-5-746-754

УДК 519.87

\title{
Control of Stochastic Processes that Proceeds in the Limited Area
}

\author{
Alexander V. Medvedev \\ Eugene D. Mikhov* \\ Siberian Federal University \\ Krasnoyarsk, Russian Federation
}

Received 16.06.2020, received in revised form 09.07.2020, accepted 06.09.2020

\begin{abstract}
Stochastic process control is considered in the paper. New types of processes (H-processes) are described. Input variables are stochastically related in H-processes. The problem of identification and control of H-processes are considered in detail. The modification of the nonparametric dual control algorithm is developed. The proposed algorithm is compared with the PID algorithm. Application of the proposed algorithm for controlling the H-process with several output variables is presented.

Keywords: nonparametric algorithms, H-prosesses, controlling.

Citation: A.V. Medvedev, E.D. Mikhov, Control of Stochastic Processes that Proceeds in the Limited Area, J. Sib. Fed. Univ. Math. Phys., 2020, 13(6), 746-754. DOI: 10.17516/1997-1397-2020-13-6-746754.
\end{abstract}

\section{Introduction}

Control of multidimensional inertialess processes is considered in the paper.

It is assumed that a controlled process has a parametric structure. In other words, in designing a control algorithm differential equation or system of equations that describes the process is known.

Often the structure of the controlled process is not completely known. In this case, before designing a control algorithm, one need to restore the structure of the controlled process.

Restoring the structure of the process is very complicated process. A control algorithm that does not require restoring of the structure of the control process is considered in the paper. In other words only the values of the input variables $\vec{u}$ and output variables $\vec{x}$ are used in the algorithm. It is assumed that some qualitative characteristics of the process are also known, such as inertia and the degree of nonlinearity of the process.

One of the features of considered processes is stochastic dependence between components of the vector of input variables $(\vec{u})$. That is why the process proceeds not in domain $\Omega(\vec{u})$ determined by the vector of input variables but in some subdomain $\Omega^{H}(x)$. Often the fact that components of input variables are interdependent is unknown. Of course, the type of relationship is also unknown.

Processes with stochastic interdependency of components of the vector of input variables are called H-processes [1].

Processes in which components of the vector of input variables $\vec{u}$ should be supplied in a certain proportion are H-processes.

Multidimensional H-processes are considered in the paper. Multidimensional H-processes include multiple output variables $\vec{x}$. For each component of the vector of output variables

*edmihov@mail.ru

(c) Siberian Federal University. All rights reserved 
$x_{j}, j=\overline{1, k}$ there is its own $\Omega^{H}\left(x_{j}\right)$, where $\mathrm{k}$ is the number of elements in the vector of output variables. In other words, the interrelation between input variables is different for each output variable $x_{j}$.

For example, consider some chemical process with two output products $(k=2)$. To obtain the first output product one should satisfy some conditions. In other words, temperature, pressure, oxygen supply, etc. must be taken into account (components of vector $\vec{u}$ ). The process proceeds when values of some input variables $\vec{u}$ satisfy some relationships. The domain where these relationships are satisfied is $\Omega^{H}\left(x_{1}\right)$.

To obtain the second output product one should satisfy some other conditions $\left(\Omega^{H}\left(x_{2}\right)\right)$. However, these conditions may be different from conditions for the first product $\left(\Omega^{H}\left(x_{1}\right) \neq \Omega^{H}\left(x_{2}\right)\right)$. Only the values of input variables that satisfy at the same time the conditions for both products allow one to obtain both products. Both products can be obtained in domain $\Omega^{H}\left(x_{12}\right)=\Omega^{H}\left(x_{1}\right) \cap \Omega^{H}\left(x_{2}\right)$.

The described above process is an example of a multidimensional H-process.

Obviously, there are many processes with this feature. Standard control algorithms (P -, PI -, PID - regulators) do not use the process domain $\Omega^{H}(x)$. That is why these control algorithms are not suitable for the processes under consideration.

Thus the need to construct new control algorithms for multidimensional H-processes is actual issue.

The simplified schematics of the considered control loop is shown in Fig. 1, where A is the considered process, $\vec{u}$ is the vector of input variables, $\vec{x}$ is the vector of output variables, $\vec{x}^{*}$ is the setting action and $\xi$ is the noise.

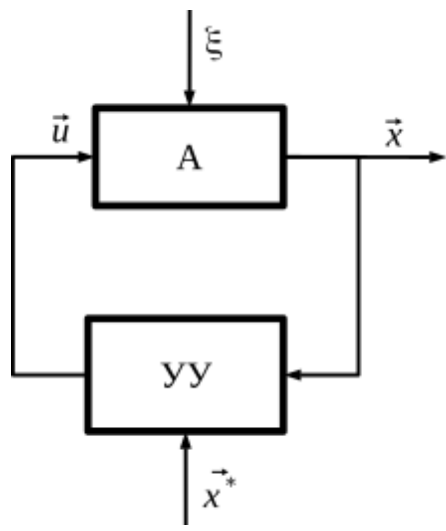

Fig. 1. Schematic representation of the control loop

The H-process does not proceed in domain $\Omega(x)$ but in some subdomain of $\Omega^{H}(x)$. A schematic representation of the multidimensional H-process is shown in Fig. 2, where $\vec{u}$ is the input vector of dimension $n$.

The process is characterized by vector of output variables $\vec{x}$ of dimension $k$. Arrows indicate the interrelation between the input variables.

It is important that the values of the components of the setting action belong to $\Omega^{H}\left(x_{j}\right), j=$ $\overline{1, k}$. There is no information on interrelation between input variables. Then it is difficult to determine the domain to which every output variable belongs.

Suppose that the process proceeds in domain $\Omega^{H}(x)$. One should find subdomain $\Omega^{H^{\prime}}(x) \in$ $\Omega^{H}(x)$ which is part of process domain (Fig. 3).

However, it can be difficult to find domain $\Omega^{H^{\prime}}(\vec{u})$ because domain $\Omega^{H}(\vec{u})$ is unknown. In addition there can be several intersections of domains $\Omega^{H}\left(x_{j}\right), j=\overline{1, k}$. Then one needs to 


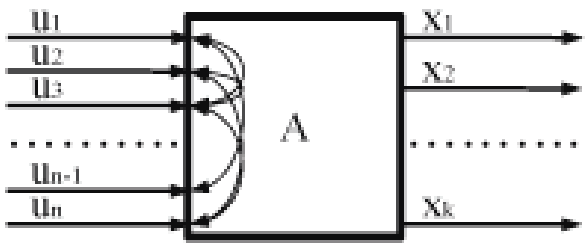

Fig. 2. Schematic representation of multidimensional H-process

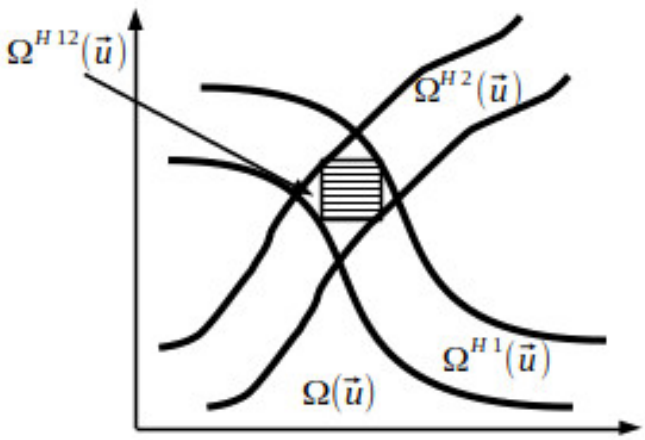

Fig. 3. Isolation of H-process flow domain

decide: what intersections should be used to control the process? Should the transition between these intersections be used in control and how the transition influences the process control?

These difficulties demonstrate that development of control algorithms for multi-dimensional H-process is the topical problem. The control algorithms should use the domain where the process proceeds and it should include some analysis of the control object. In other words, the control algorithm should be the adaptive algorithm.

The nonparametric dual control algorithm [1] is used in the paper. The nonparametric dual control algorithm is based on two methods: the dual control method developed by A. A. Feldbaum [2] and the nonparametric regression function estimation method [4]. The nonparametric dual control algorithm was developed by A.V. Medvedev [3].

\section{The nonparametric regression function estimation}

Let us consider statically independent observations of two random variables $(x, y)=$ $\left(x_{1}, y_{1}\right), \ldots,\left(x_{n}, y_{n}\right)$ that are distributed with unknown frequency function $P(x, y)$.

Let us assume that $p(x)>0 \forall x \in \Omega(x)$. To approximate the unknown stochastic relationship between $y$ and $x$ the regression is often used [4]:

$$
y=f(x)=\left(\int_{\Omega(y)} P(x, y) d y\right)^{-1}\left(y \int_{\Omega(y)} P(x, y) d y\right)
$$

Nonparametric estimation of relation (1) is 


$$
\hat{y}=\hat{f}(x)=\left(\sum_{i=1}^{n} \Phi\left(\frac{x-x_{i}}{C_{n}}\right)\right)^{-1} \sum_{i=1}^{n} y_{i} \Phi\left(C_{n}^{-1}\left(x-x_{i}\right)\right)
$$

When $x=\left(x_{1}, \ldots, x_{k}\right)$ and $y=\left(y_{1}, \ldots, y_{k}\right)$ are vectors relation (2) becomes

$$
\hat{y}_{d}=\frac{\sum_{i=1}^{n} y_{i}^{d} \prod_{j=k}^{k} \Phi\left(C_{j}^{-1}(n)\left(x^{j}-x_{i}^{j}\right)\right)}{\sum_{i=1}^{n} \prod_{j=k}^{k} \Phi\left(C_{j}^{-1}(n)\left(x^{j}-x_{i}^{j}\right)\right)}, \quad d=\overline{1, k}
$$

The nonparametric estimate of the regression curve is convergent, i.e.,

$$
\begin{gathered}
\lim _{n \rightarrow \infty} M\left(\left(f(x)-f_{n}(x)\right)^{2}\right)=0, \forall x \in \Omega(x) \\
\lim _{n \rightarrow \infty} M(f(x))=f(x), \forall x \in \Omega(x) .
\end{gathered}
$$

Information on the parametric structure of the object is not needed for the noparametric estimate of regression function.

\section{Nonparametric dual control}

In the case when control algorithm includes control and investigation of the system, it is called dual control algorithm.

Dual control algorithm was developed by A. A. Feldbaum. The nonparametric dual control algorithm is

$$
u_{s+1}=u_{s}^{*}+\delta u_{s+1},
$$

where $u_{s}^{*}$ is "knowledge" of the object, $\delta u_{s+1}$ is "learning" search steps (in the classic form of nonparametric dual control algorithm) and $\delta u_{s+1}$ is

$$
\delta u_{s+1}=\xi\left(x_{s+1}^{*}-x_{s}\right) .
$$

Using the noparametric estimate of the regression function $\left(x_{i}, u_{i}\right), i=\overline{1, s}$, we obtain the estimate of the object $\hat{x}=\hat{f}(\vec{u})$ as

$$
\hat{x}(u)=\frac{\sum_{i=1}^{n} x_{i} \Phi\left(\frac{u-u_{i}}{c_{s}}\right)}{\sum_{i=1}^{n} \Phi\left(\frac{u-u_{i}}{c_{s}}\right)} .
$$

Here bell-shaped functions $\Phi(\cdot)$ and smooth coefficient $c_{s}$ satisfy convergence condition, $u=$ $f^{-1}(x)$, where $f^{-1}(x)$ is the inverse of $f(u)$, and $u_{s}^{*}$ is

$$
u_{s}^{*}=\frac{\sum_{i=1}^{n} u_{i} \Phi\left(\frac{\Phi\left(x^{*}-x_{i}\right)}{c_{s}}\right)}{\sum_{i=1}^{n} \frac{\Phi\left(x^{*}-x_{i}\right)}{c_{s}}},
$$

where $x^{*}$ is the setting action.

At the beginning of process control, second component $\delta u_{s+1}$ is more important component of control. This is the time of active investigating of the dual control system. This stage begins with receiving of the first values of the input and output variables. The first component $\left(u_{s *}\right)$ becomes more important component of control after stage of active investigating. Thus, there are stage of object investigation and stage of action in the process of dual control. 


\section{Modification of nonparametric dual control algorithm for multidimensional $\mathrm{H}$-processes}

In multidimensional H-process control the action cannot be arbitrarily specified as it is considered in control theory. This is due to the fact that it is possible to set a vector of action such that $\prod i=1^{k} \Omega_{i}^{H}\left(\vec{x}^{*}\right)=\varnothing$. In other words, this action is not achievable for all components of vector $\vec{x}^{*}$ at the same time. That is why it is important to set $\vec{x}^{*} \in \prod_{i=1}^{k} \Omega_{i}^{H}\left(\vec{x}^{*}\right)$, i.e., define $x_{1}^{*}, x_{2}^{*}, \ldots, x_{k}^{*}$.

We propose the following method:

1. Calculate the value $\sum_{i=1}^{s} \prod_{j=1}^{k} \Phi\left(\frac{x_{j}^{*}-x_{i j}}{c_{s j}}\right)$, where $\vec{x}^{*}$ is the action and $s$ is the size of sample observations.

2. If the calculated value $\sum_{i=1}^{s} \prod_{j=1}^{k} \Phi\left(\frac{x_{j}^{*}-x_{i j}}{c_{s j}}\right)$ is not equal to zero then the action is achievable otherwise the action may not be achieved.

Let us note that in nonparametric dual control the calculation of the search step $\delta \vec{u}_{s+1}$ is performed with the use of (7). In the case of H-process with several output variables the described method for calculating the search step $\delta \vec{u}_{s+1}$ is not suitable because the input action must belong to $\prod_{j=1}^{k} \Omega^{H}(\vec{u})$.

Taking this into account, we propose to use an algorithm with punishment to calculate $\delta \vec{u}_{s+1}$.

This method determines the reachability of the action and it can be described as follows

1) $\vec{u}_{s}\left(\vec{x}^{*}\right)$ is calculated;

2) a random vector $\delta \vec{u}_{s+1}$ is generated;

3) $\vec{u}_{s+1}\left(x^{*}\right)$ is calculated;

4) if $\vec{u}_{s+1} \in \Omega^{H}(\vec{u})$ then $\vec{u}_{s+1}$ is used as a control action otherwise we return to step 2;

5) if $\sum_{i=1}^{k}\left|x_{i, s+1}-x_{i}^{*}\right|<\sum_{i=1}^{k}\left|x_{i, s}-x_{i}^{*}\right|$ then $\delta \vec{u}_{s+1}$ is used as the next value of the search step $\delta \vec{u}_{s+2}$ otherwise the random vector $\delta \vec{u}_{s+2}$ is generated;

6) go back to step 1.

The length of the vector $\delta \vec{u}$ is $m\left|x^{*}-x_{s}\right|$, where $m$ is the preassigned coefficient.

\section{Results of computer simulation}

Examples of control of the inertialess process and H-process are presented. Standard regulator and regulator on the basis of the nonparametric algorithm of dual control are used in simulations.

Firstly, the PID regulator is compared with the modified algorithm of nonparametric dual control. Secondly, the nonparametric dual control algorithm is used to control the multidimensional H-process.

The simulated multidimensional process is defined as follows

$$
\begin{gathered}
x_{1}(\vec{u})=f\left(u_{1}, u_{2}\right)=u_{1} * 3 * \sin \left(u_{2}\right)+7+\xi_{1}, \\
x_{2}(\vec{u})=f\left(u_{1}, u_{2}\right)=4 *\left(u_{1}\right)+u_{2}+\xi_{2} .
\end{gathered}
$$

Let us note that structure of the object is not used in the algorithm. It is used only to generate a sample of observations.

Next the result of control based on the PID regulator is presented. 
The result of control over $x_{1}$ is shown in the upper part of Fig. 4. The result of control over $x_{2}$ is shown in the lower part of the figure. The value of the action is marked by the dashed line. The iteration number is shown on the abscissa.
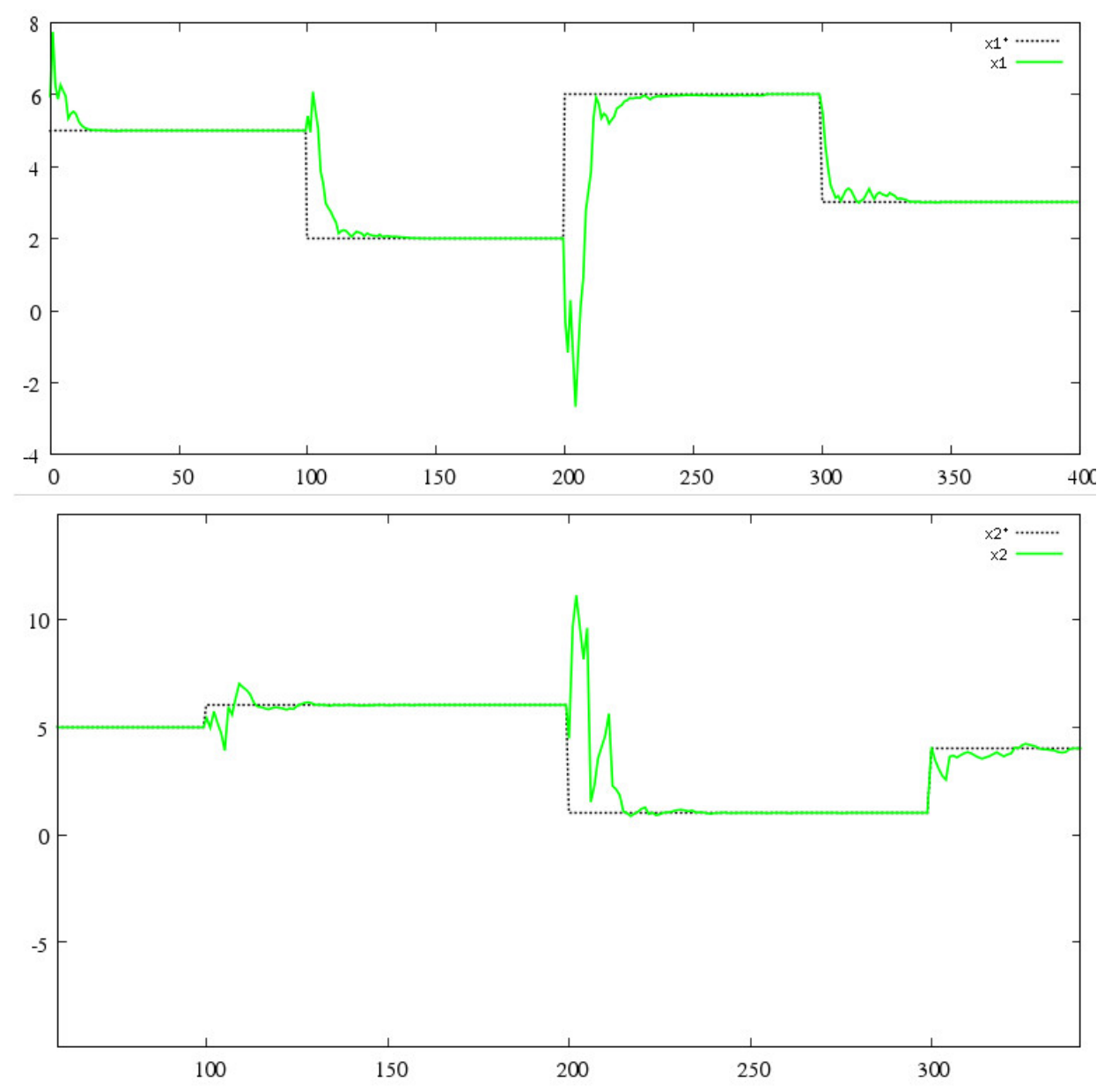

Fig. 4. Result of the PID control

Results presented in figure 4 demonstrate that PID algorithm successfully controls object (10). This algorithm is not adaptive, and it does not include training. This means that algorithm controls the process without using a sample of observations to improve its characteristics. That is why the control efficiency did not increase.

Nonparametric dual control algorithm is used to control process (10) in the next experiment.

Unlike the PID algorithm used in the previous experiment (Fig. 5) this algorithm includes training. This is confirmed by the fact that after training the control is more efficient.

Next we consider a multidimensional inertialess H-process. There is a stochastic relationship between input variables in the H-process.

The process is described by the system of equations (10). The relationship between input variables is described by the following equations

$$
u_{2}=u_{1} * 5-3+\xi_{1}, \xi_{1} \in(-0.4 ; 0.4)
$$



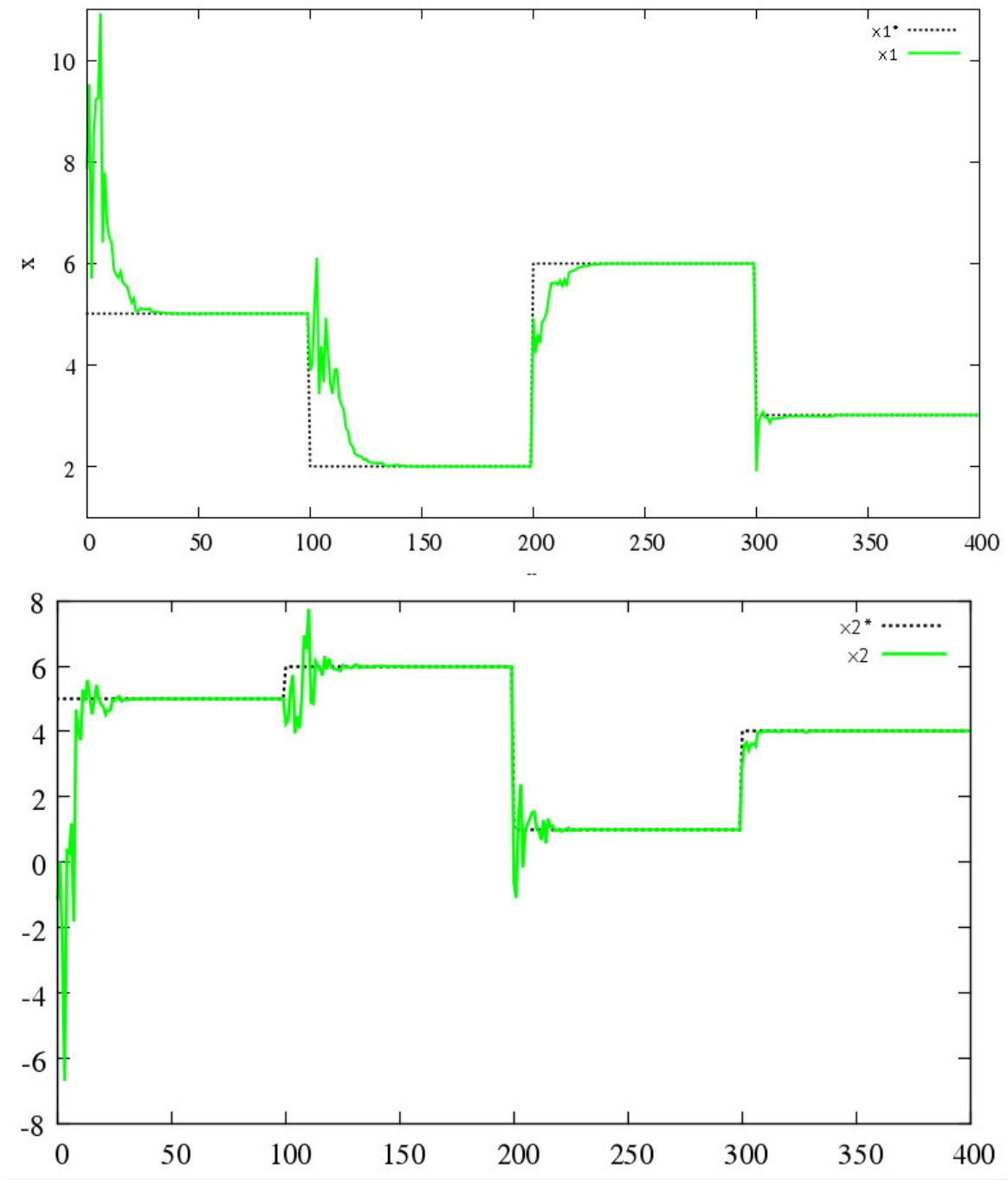

Fig. 5. Result of nonparametric dual control

$$
u_{1}=u_{2} * 3+1+\xi_{2}, \xi_{2} \in(-0.6 ; 0.6) .
$$

Variables $\xi_{1}, \xi_{2}$ characterize the "width" of domains $\Omega^{H 1}(\vec{u})$ and $\Omega^{H 2}(\vec{u})$, respectively.

It is difficult to control this H-process using the PID algorithm because this algorithm does not take into account domain $\left(\Omega^{H 1}(\vec{u}), \Omega^{H 2}(\vec{u})\right)$. This is important in the case of the H-process.

The H-process under consideration is controlled with the use of the modified nonparametric dual control algorithm (Fig. 6).

The modification of the nonparametric dual control algorithm can be successfully applied to control the multidimensional H-process. It is demonstrated in Fig. 6. The nonparametric dual control algorithm is adaptive.

Therefore, after training, the proposed modification more effectively controls the process. 

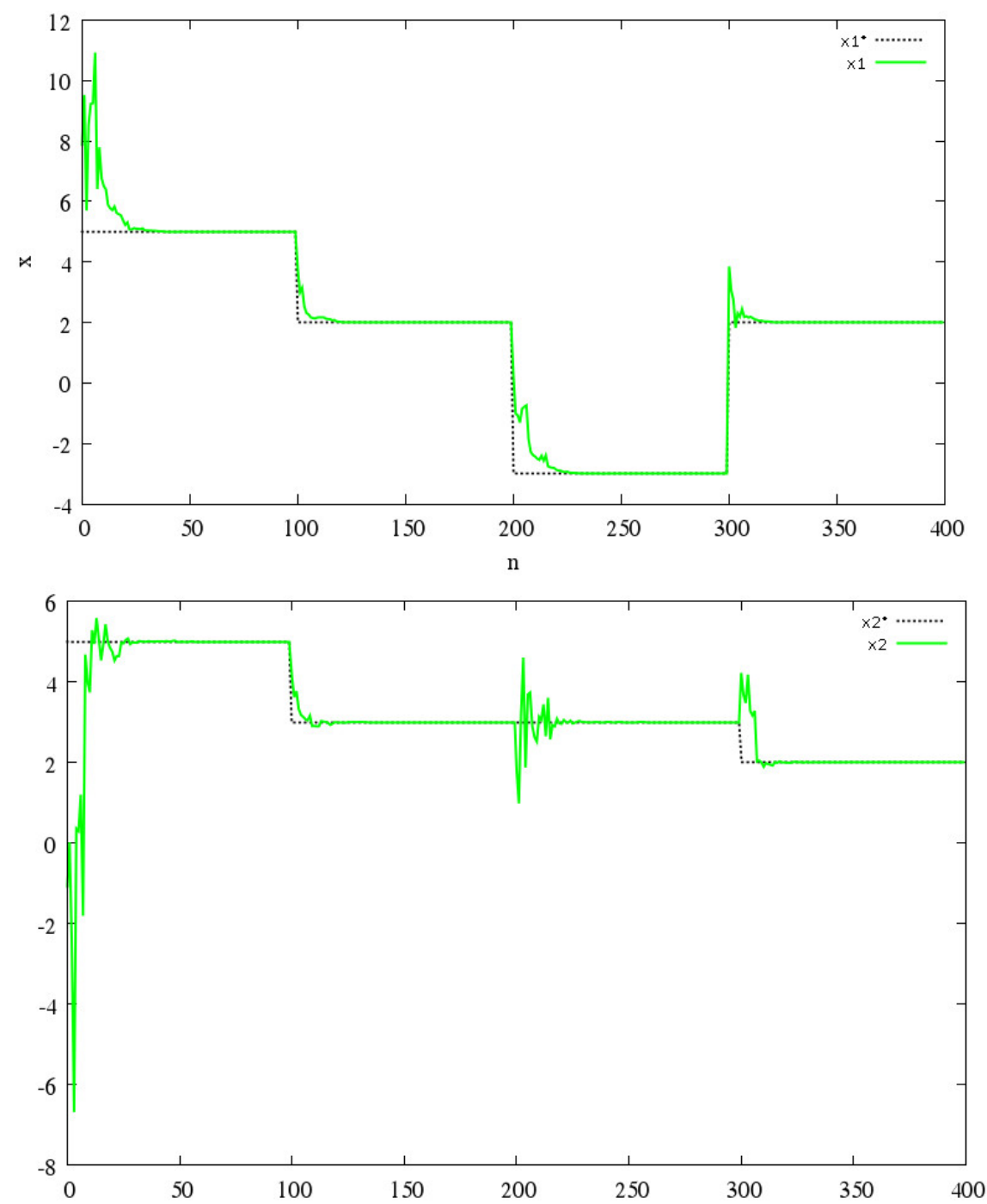

Fig. 6. Result of control with the modified nonparametric dual control algorithm

\section{Conclusion}

A modification of the nonparametric dual control algorithm was proposed. A feature of the proposed modification is a new choice of the search step which takes into account the domain of the process. The proposed modification was applied to control multidimensional inertialess processes with interdependent input variables.

The modified nonparametric dual control algorithm and the PID algorithm was compared. It was demonstrated that the modified nonparametric dual control algorithm is adaptive. After training this algorithm controls the process more effectively than the PID algorithm. The modification of nonparametric dual control was applied to a multidimensional inertialess H-process. The proposed algorithm successfully controls the multidimensional inertialess H-process.

This work was financially supported by the Ministry of Science and Higher Education of the Russian Federation under the project "Creation of a production of earth stations of advanced 
satellite communications systems to ensure the coherence of hard, northern and Arctic territory of Russian Federation", implemented with the participation of the Siberian Federal University (agreement number 075-11-2019-078 dated 13.12.2019).

\title{
References
}

[1] A.V.Medvedev, Nonparametric adaptation systems, Novosibirsk, Nauka, 1983 (in Russian).

[2] A.A.Feldbaum, Fundamentals of the theory of optimal automatic systems, Moscow, Fizmatgiz, 1963 (in Russian).

[3] A.Medvedev, Theory of nonparametric systems. Modeling, Bulletin of SibSAU, 30(2010), no. 4, 4-9 (in Russian).

[4] P.Eykhoff, Fundamentals of identification of control systems, Moscow, Mir, 1975.

\section{Управление стохастическими процессами с ограниченной областью протекания}

\section{Александр В. Медведев}

Евгений Д. Михов

Сибирский федеральный университет

Красноярск, Российская Федерация

\begin{abstract}
Аннотация. В статье рассматриваются вопросы управления стохастическими процессами. Описаны новые виды процессов (Н-процессы) в которых имеется стохастическая зависимость между входными переменными. Описываются проблемы, возникающие при решении задачи идентификации и управления. Для решения этих проблем при решении задачи управления был предложена модификация алгоритма непараметрического дуального управления. Проведены эксперименты, в которых предложенная модификация алгоритма сравнивается по эффективности с ПИД регулятором. В конце статьи представлен эксперимент по управлению Н-процессом с несколькими выходными переменными, при помощи разработанного алгоритма.
\end{abstract}

Ключевые слова: непараметрические алгоритмы, Н-процессы, управление. 